\title{
UNIVALENCE FOR CONVOLUTIONS
}

\author{
HERB SILVERMAN
}

\author{
Department of Mathematics \\ University of Charleston \\ Charleston, SC 29424
}

(Received May 10, 1994)

\begin{abstract}
The radius of univalence is found for the convolution $f * g$ of functions $f \in S$ (normalized univalent functions) and $g \in C$ (close-to-convex functions). A lower bound for the radius of univalence is also determined when $f$ and $g$ range over all of $S$. Finally, a characterization of $C$ provides an inclusion relationship.
\end{abstract}

KEY WORDS AND PHRASES. Univalent, convolution. 1991 AMS SUBJECT CLASSIFICATION CODE(S). Primary 30C45, Secondary 30C50.

\section{INTRODUCTION.}

Denote by $S$ the family consisting of functions $f(z)=z+\cdots$ that are analytic and univalent in $\Delta=\{z:|z|<1\}$ and by $K, S^{*}$, and $C$ the subfamilies of functions that are, respectively, convex, starlike, and close-to-convex in $\Delta$. It is well known that $K \subset S^{*} \subset C \subset S$. The convolution of two power series

$$
f(z)=\sum_{n=0}^{\infty} a_{n} z^{n} \text { and } g(z)=\sum_{n=0}^{\infty} b_{n} z^{n}
$$

is defined as the power series

$$
f(z)=\sum_{n=0}^{\infty} a_{n} b_{n} z^{n}
$$

The Koebe function $k(z)=z /(1-z)^{2}$ often plays an extremal role in the family $S$. This enables us to show it to be extreme in many convolution problems. For example, the modulus of the $n$th coefficient for $f * g, f$ and $g$ in $S$, is $n^{2}$ and is attained when $f=g=k$. Similarly, $|f * g|$ takes its maximum and minimum on the circle $|z|=r$ when $f=g=k$.

A question was raised in [4] as to whether

$$
\underset{|z|=r}{\min } \operatorname{Re} \frac{(f * g)(z)}{z}=\underset{|z|=r}{\min } \operatorname{Re} \frac{(f * k)(z)}{z}=\underset{|z|=r}{\min } \operatorname{Ref}
$$

when $f$ and $g$ are taken over all of $S$. The classical rotation theorem for $f \in S$ leads to the sharp result that $\operatorname{Ref}^{\prime}(z) \geq 0$ when $|z| \leq \sin (\pi / 8)$. This was generalized in [4] to $\operatorname{Re} \frac{(f * g)(z)}{z} \geq 0$ for $|z| \leq \sin (\pi / 8)$ when $f \in S$ and $g \in S^{*}$, but could not be extended to $g \in S$ or even to $g \in C$. In particular, functions $f, g \in C$ were found for which $\operatorname{Re} \frac{(f * g)(z)}{z}<0$ at some point $z_{0},\left|z_{0}\right|<\sin (\pi / 8)$. 
In this note, we investigate the radius of univalence for $f * g, f$ and $g$ in $S$ For $f \in S$ and $g=k$, the Koebe function, $f * g$ is univalent in the disk $|z|<2-\sqrt{3}$ We prove that $g=k$ can be replaced by any $g \in C$, but we cannot settle if this extends to arbitrary $g \in S$. We do show, however, that $f * g$ is univalent for at least $|z|<.8(2-\sqrt{3})$

2. MAIN RESULTS.

THEOREM 1. If $f \in S$ and $g \in C$, then $f * g$ is univalent in $|z|<2-\sqrt{3}$. The result is sharp

PROOF. It is well known that $f$ is convex in $|z|<r$ if and only if $z f^{\prime}$ is starlike in $|z|<r$ and that the radius of convexity of $S$ is $2-\sqrt{3}$. Thus, $f * k=z f^{\prime}$ has radius of starlikeness (and hence radius of univalence) at least $2-\sqrt{3}$, the radius of convexity for $f \in S$ Since

$$
(k * k)^{\prime}=\left(z k^{\prime}\right)^{\prime}=\frac{1+4 z+z^{2}}{(1-z)^{4}}=0 \text { at } z=-(2-\sqrt{3}),
$$

the radius of univalence of $f * g$ for $f, g \in S$ can be no greater than $r=2-\sqrt{3}$.

When $f \in S$, we have $f(a z) / a \in K$ for $a=2-\sqrt{3}$. Hence, by a theorem of Ruscheweyh and Sheil-Small [3], if $f \in S$ and $g \in C$ then

$$
\frac{f(a z)}{a} * g(z) \in C \subset S
$$

Thus, $f * g$ is univalent for $|z|<2-\sqrt{3}$, and the proof is complete.

In our next theorem, we replace $C$ with $S$ in the hypothesis and this leads to a weaker conclusion.

THEOREM 2. Denote by $r_{0}$ the largest value for which $f * g$ is univalent in $|z|<r_{0}$ for all $f, g \in S$. Then $.8(2-\sqrt{3})<r_{0} \leq 2-\sqrt{3}$.

PROOF. The upper bound was found in Theorem 1. Krzyz [1] determined the radius of close-to-convexity for $S$ to be $t_{0}=0.80+$. Since $f(a z) / a \in K, a=2-\sqrt{3}$, and $g\left(t_{0} z\right) / t_{0} \in C$, we have from the Ruscheweyh and Sheil-Small theorem [3] that $\frac{f(a z)}{a} * \frac{g\left(t_{0} z\right)}{t_{0}} \in C$, which shows that $f * g$ is univalent for $|z|<t_{0}(2-\sqrt{3})$. This furnishes us with the lower bound, and the proof is complete.

Though we are unable to prove that $r_{0}=2-\sqrt{3}$ in Theorem 2 , the lower bound on $r_{0}$ most certainly can be improved. Ruscheweyh defined the family $M$ consisting of normalized functions $f$ by

$$
M=\left\{f: f * g \neq 0 ; g \in S^{*}, 0<|z|<1\right\}
$$

He proved the proper inclusions $C \subset M \subset S$ and that $f * g \in M$ for $f \in K$ and $g \in M$ [2]. Hence, if $t_{1}$ is the largest value for which $g\left(t_{1} z\right) / t_{1} \in M$ when $g=S$, methods identical to those of Theorem 2 show that $f * g$ is univalent in $|z|<t_{1}(2-\sqrt{3})$ for $f, g \in S$. Unfortunately the value of $t_{1}$, the radius of " $M$-ness" for $S$, is unknown.

\section{A CHARACTERIZATION OF $C$.}

The inclusion $C \subset M$ is not obvious and was proved by Ruscheweyh using his duality principle [2]. Our final result is a characterization of $C$ that leads to a more elementary proof that $C \subset M$. We make use of a result found in [3].

LEMMA 3. If $\phi \in K, \Psi \in S^{*}$, and $F$ is analytic with $R e F>0$ for $z \in \Delta$, then

$$
R e \frac{\phi * F \Psi}{\phi * \Psi}>0
$$


THEOREM 3. A function $f \in C$ if and only if to each $g \in S^{*}$ we may associate an $h \in S^{*}$ for which $\operatorname{Re} \frac{f * g}{h}>0, z \in \Delta$.

PROOF. To show that the condition is sufficient for $f$ to be in $C$, we choose $g(z)=z /(1-z)^{2} \in S^{*}$. Then $\operatorname{Re} \frac{f * g}{h}=R e \frac{z f^{\prime}}{h}>0$, which means that $f \in C$.

On the other hand, if $f \in C$ we can find a $\Psi=S^{*}$ for which $R e z f^{\prime} / \Psi>0$ Set $F(z)=z f^{\prime}(z) / \Psi(z)$. Then for $g \in S^{*}$ there corresponds $\phi \in K$ such that $z \phi^{\prime}=g$. Note that $f * g=z f^{\prime} * \phi=\phi * F \Psi$ and that $h=\phi * \Psi \in S^{*}$. By Lemma A,

$$
R e \frac{\phi * F \Psi}{\phi * \Psi}=R e \frac{f * g}{h}>0,
$$

and the proof is complete

COROLLARY. $C \subset M$

PROOF. Since $R e \frac{f * g}{h}>0 \Rightarrow f * g \neq 0$, the result follows from Theorem 3 .

\section{REFERENCES}

1. KRZYZ, J., The radius of close-to-convexity within the family of univalent functions, Bull. Acad. Polon. Sci. 12 (1964), 25-28.

2. RUSCHEWEYH, ST., Convolutions in geometric function theory, Les Presses De $L^{\prime}$ Université De Montréal, Montreal, Canada, 1982.

3. RUSCHEWEYH, ST. and SHEIL-SMALL, T., Hadamard products of schlicht functions and the Pólya-Schoenberg conjecture, Comment. Math. Helv. 48 (1973), 119-135.

4. SILVERMAN, H., Radii problems for the convolution of univalent functions, Houston J. Math. 19 (1993), 45-50. 


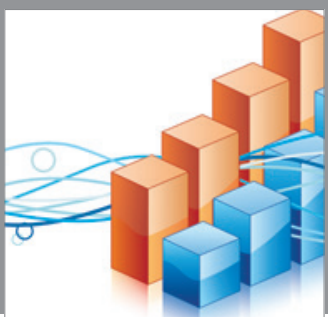

Advances in

Operations Research

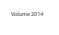

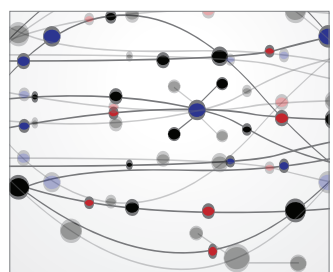

\section{The Scientific} World Journal
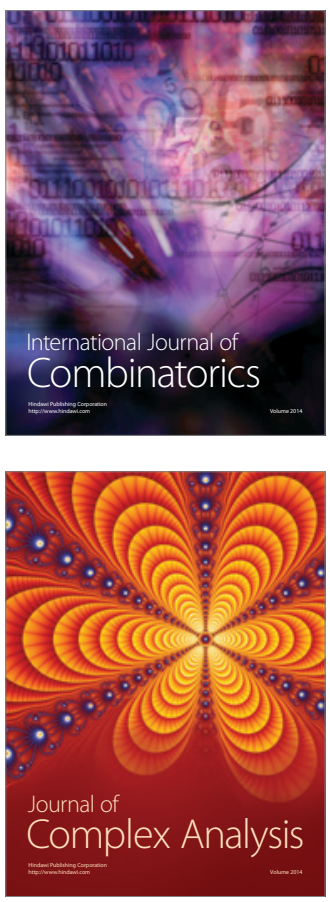

International Journal of

Mathematics and

Mathematical

Sciences
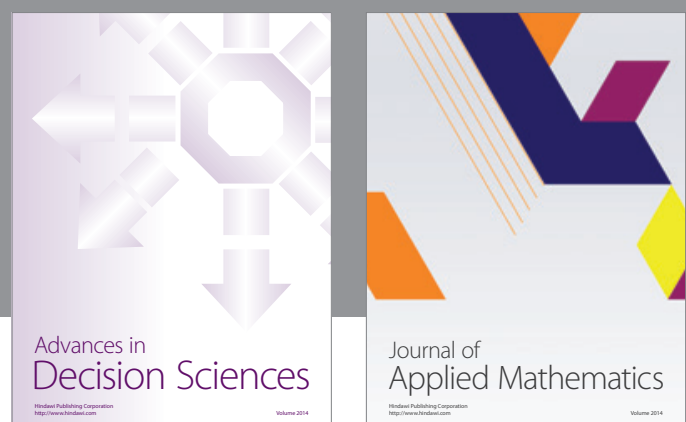

Journal of

Applied Mathematics
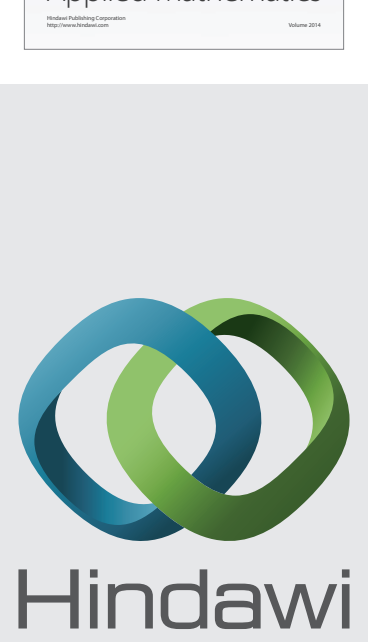

Submit your manuscripts at http://www.hindawi.com
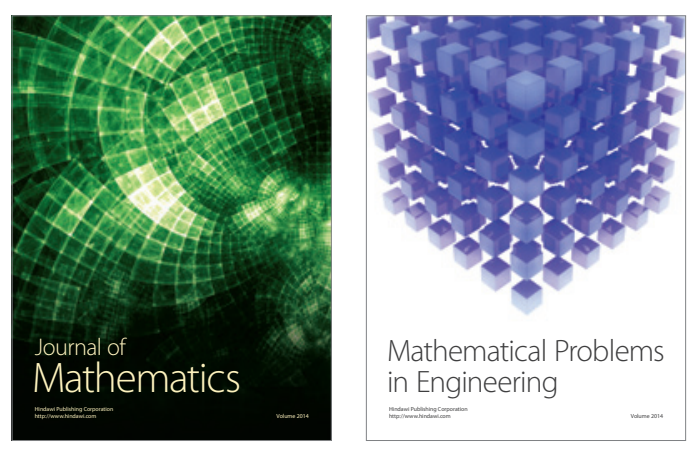

Mathematical Problems in Engineering
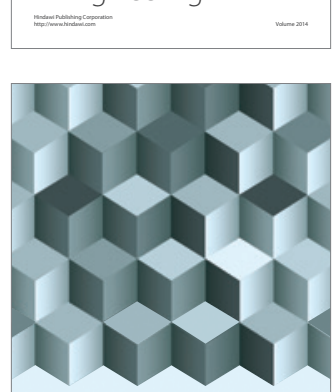

Journal of

Function Spaces
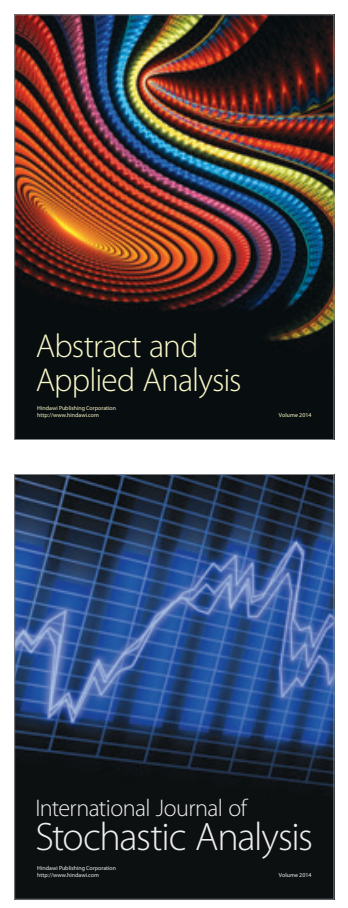

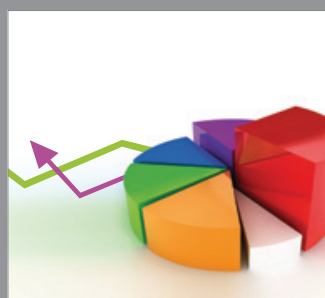

ournal of

Probability and Statistics

Promensencen
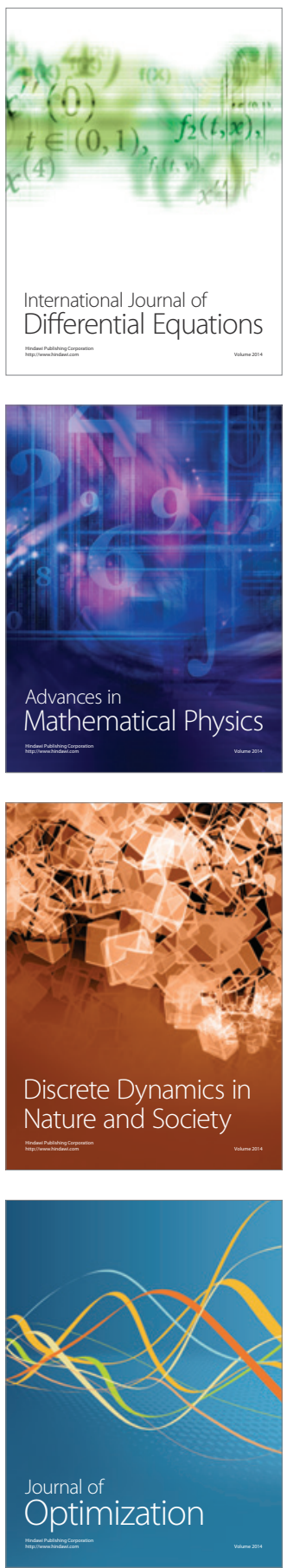